\title{
Envisager le milieu littéraire avec une perspective féminine : le cas de Jeannette Boulizon
}

\section{The Literary Milieu from a Feminist Perspective: The Case of} Jeannette Boulizon

\section{Acercamiento al medio literario desde una perspectiva femenina: el caso de Jeannette Boulizon}

\section{Fanie St-Laurent}

Volume 56, numéro 4, octobre-décembre 2010

Parcours et trajectoires de médiateurs culturels

URI : https://id.erudit.org/iderudit/1029040ar

DOI : https://doi.org/10.7202/1029040ar

Aller au sommaire du numéro

Éditeur(s)

Association pour l'avancement des sciences et des techniques de la documentation (ASTED)

ISSN

0315-2340 (imprimé)

2291-8949 (numérique)

Découvrir la revue

Citer cet article

St-Laurent, F. (2010). Envisager le milieu littéraire avec une perspective féminine : le cas de Jeannette Boulizon. Documentation et bibliothèques, 56(4), 162-168. https://doi.org/10.7202/1029040ar
Résumé de l'article

Jeannette Boulizon émigre au Canada en 1938 et fait partie de l'équipe de professeurs qui fonde le Collège Stanislas de Montréal. Elle s'intègre rapidement à la société canadienne-française et devient à la fois professeure au primaire et membre d'organisations religieuses, éducatives et culturelles. Son parcours pluridirectionnel l'a amenée soit à élargir sa culture personnelle, soit à la partager. Il semble que son statut d'immigrante française scolarisée et active sur le marché du travail lui aura donné une crédibilité peu habituelle avant les années 1970.
Tous droits réservés (C) Association pour l'avancement des sciences et des techniques de la documentation (ASTED), 2010
Ce document est protégé par la loi sur le droit d'auteur. L’utilisation des services d’Érudit (y compris la reproduction) est assujettie à sa politique d'utilisation que vous pouvez consulter en ligne. 


\title{
Envisager le milieu littéraire avec une perspective féminine : le cas de Jeannette Boulizon
}

\author{
FANIE ST-LAURENT \\ Doctorante, Groupe de recherches et d'études sur le livre au Québec (GRÉLQ) \\ Université de Sherbrooke \\ Fanie.St-Laurent@USherbrooke.ca
}

RÉSUMÉ | ABSTRACTS | RESUMEN
Jeannette Boulizon émigre au Canada en 1938 et fait partie de
l'équipe de professeurs qui fonde le Collège Stanislas de Montréal.
Elle s'intègre rapidement à la société canadienne-française et
devient à la fois professeure au primaire et membre d'organisa-
tions religieuses, éducatives et culturelles. Son parcours pluridirec-
tionnel l'a amenée soit à élargir sa culture personnelle, soit à la
partager. Il semble que son statut d'immigrante française scola-
risée et active sur le marché du travail lui aura donné une crédibi-
lité peu habituelle avant les années 197o.
$\quad$ The Literary Milieu from a Feminist Perspective:
$\quad$ The Case of Jeannette Boulizon
Jeannette Boulizon immigrated to Canada in 1938 and was one of
the founding members of the Collège Saint-Stanislas in Montréal.
She became quickly involved in French-Canadian life, teaching in
a primary school and participating actively in various religious,
educational and cultural organisations. Her professional life took
several directions, broadening her cultural outlook and sharing it
with others. It seems that the fact that she was an educated French
immigrant, active in the workforce gave her unusual credibility in
the period prior to the 197os.
Acercamiento al medio literario desde una perspectiva
femenina : el caso de Jeannette Boulizon
Jeannette Boulizon emigra a Canadá en 1938 y forma parte del
equipo de profesores fundadores del colegio Stanislas de Montreal.
Se adapta rápidamente a la sociedad canadiense-francesa y se
desempeña como maestra primaria mientras participa de organi-
zaciones religiosas, educativas y culturales. Su trayectoria multi-
direccional la lleva a ampliar y a compartir su cultura personal.
Su situación de inmigrante francesa educada y su participación
en el mercado laboral le habrín otorgado una credibilidad poco
común antes de la década de los setenta.

L ES RECHERCHES RÉCENTES EN HISTOIRE du livre ont fait sortir de l'ombre de nombreux agents du circuit de communication du livre, pour reprendre les termes de Robert Darnton (1992). Les travaux d'Éric Leroux sur l'imprimerie (2005), de Jacques Michon sur l'édition littéraire (1999 et 2004) et de Fernande Roy sur la librairie (2000) ont mis au jour, pour le Québec, le rôle de certains métiers et de certains acteurs surtout des hommes. Certains ouvrages montrent aussi la place qu'ont occupée les femmes dans ces métiers. Par exemple, Jef Tombeur s'est intéressé aux femmes et aux métiers du livre dans les pays anglophones et francophones de l'Europe (2004) et Roméo Arbour a répertorié en un dictionnaire les femmes libraires en France entre 1470 et 1870 (2003). Les Gender Studies ont, quant à elles, développé des études sur les lectures des femmes. Julia Bettinotti s'est intéressée à la question de la lecture du roman sentimental (1990) alors que Sophie Montreuil a fouillé les pratiques individuelles et collectives de lecture au $\mathrm{XIX}^{\mathrm{e}}$ siècle, notamment avec le cas de Joséphine Marchand-Dandurand et de son CEuvre des livres gratuits (2003). De plus, sous la direction de Monique Lebrun, l'ouvrage sur les pratiques de lecture des adolescents québécois a démontré qu'en 1980 comme en 1994, les filles lisent bien davantage que les garçons (2004). Enfin, l'histoire littéraire québécoise a reconnu le travail de quelques écrivaines depuis Marie de l'Incarnation jusqu'à Laure Conan, Germaine Guèvremont, Gabrielle Roy, Anne Hébert, Rina Lasnier et Marie-Claire Blais. À leur époque, ces femmes qui exercent le métier d'auteures étaient peu nombreuses à livrer leur vision du monde.

Cette façon d'envisager l'histoire du livre avec des catégories masculines prédéfinies (imprimerie, librairie, édition, lecture, écriture) empêche de considérer d'autres agents qui ont pu jouer un rôle actif dans la vie littéraire et culturelle. Cette lacune est soutenue par la croyance, vieille et tenace, selon laquelle les femmes ont peu ou prou influencé l'histoire du livre, si ce n'est qu'en amont, sur le plan de la production, en tant quauteures. Or s'il est un domaine où elles ont exercé de l'influence dans l'histoire culturelle, c'est bien à travers l'action souvent souterraine, certes - qu'elles ont mené au sein 
de plusieurs réseaux, dont celui du livre. Cest donc en observant l'histoire du livre avec une perspective féminine et notamment à travers létude des regroupements, que l'on comprend l'importance de ces individus. Mes recherches doctorales sur la Société détude et de conférences, ses membres et ses activités, des années 1930 aux années 2000, me permettent d'amorcer cette réflexion.

Le présent article vise à montrer qu'au-delà des apparences, l'influence des femmes dans le milieu du livre est importante et tangible. Mais pour en saisir tout l'empan, il importe détudier les réseaux dans lesquels elles déploient leurs énergies. Je veux donc m’arrêter à l'exemple de Jeannette Boulizon, membre de la Société détude et de conférences, regroupement qui participe activement à la vie littéraire dès les années 1930. En effet, en 1936 parait dans l'Almanach de la langue française, un article sur les Canadiennes françaises et les lettres, sous la direction d'Albert Lévesque et de Pierre Dagenais. On y retrouve une liste de 20 femmes de lettres du Canada français, des romancières, des poètes, des journalistes comme Jovette Bernier, Marie-Claire Daveluy, Françoise Gaudet, Blanche Lamontagne, Maxine, Odette Oligny, Simone Routier et Éva Senécal. Les portraits de trois des quatre premières présidentes de la Société détude et de conférences, Annette Doré, Rita Gariépy et Yvonne Letellier de Saint-Just, y figurent. Même si elles nont jamais publié de livres, les dirigeantes de la Société détude et de conférences sont donc reconnues par le milieu du livre, elles exercent une certaine influence et sont des vecteurs de diffusion du livre au Québec.

\section{Trajectoire d'une femme du livre}

Jeannette Boulizon, née Jeanne Chobert à Paris en 1915, émigre au Canada en 1938, lannée de son mariage. Elle fait partie, avec son mari Guy Boulizon, de l'équipe de professeurs qui a fondé le Collège Stanislas de Montréal, une institution qui offre un programme d'enseignement français et catholique dans la tradition de Stanislas de Paris. Issue de la haute bourgeoisie française - Jeannette est larrière-petite-fille de larchitecte de Napoléon -, elle surprend ses contemporains français lorsqu'elle leur annonce son départ pour le Canada, d'autant plus qu'elle quitte sa terre natale pour occuper un emploi, ce qui ne correspond pas aux coutumes de son rang social. Devant l'imminence de la guerre, sa famille l'encourage à s'expatrier. Seule femme dans le corps professoral, elle sent que sa présence étonne, dérange même. Dans une allocution prononcée à loccasion du $60^{\mathrm{e}}$ anniversaire de l'Institution en 1999, elle affirmait qu' " [...] on n'admettait pas que des laïcs - et même une fermme! - puissent enseigner dans un collège de garçons " (Boulizon, 1999).

Dans le paysage scolaire québécois, larrivée du Collège Stanislas représente une révolution, ou du moins une menace certaine pour les collèges classiques traditionnels. Dans son ouvrage sur les collèges classiques au Canada français, Claude Galarneau présente rapidement
Les dirigeantes de la Société d'étude et de conférences sont donc reconnues par le milieu du livre, elles exercent une certaine influence et sont des vecteurs
de diffusion du livre au Québec.

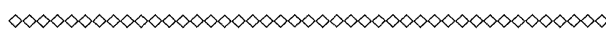

les efforts du sénateur Raoul Dandurand pour doter Montréal d'un lycée français : "Au lieu d'avoir un lycée français, Outremont se retrouve avec un collège dirigé par des prêtres français » (Galarneau, 1978 : 287), ce qui n'est pas tout à fait exact si lon tient compte du couple Boulizon, d'André Champoux, de Pierre Ricour et de René Lescop qui font partie des premiers professeurs laïques du Collège. Détentrice d'un baccalauréat, Jeannette Boulizon a effectué un stage au Collège Stanislas de Paris, puis a enseigné à celui de Montréal situé sur la rue Rockland dès septembre 1938, et, à la fin des années 1940, elle enseigne au Collège Marie-de-France, le pendant féminin de Stanislas.

Lavidité de savoir de Jeannette Boulizon la porte à aller au-delà du baccalauréat obtenu en France. Elle complète une maitrise en phonétique expérimentale à l'Université de Montréal en 1945, puis un baccalauréat en théologie en 1974, au moment de sa retraite. Ses habitudes familiales et sociales en surprennent plus d'un; notamment parce quelle conduit une voiture (tandis que son mari fait la cuisine) et qu'elle voyage seule avec ses enfants tous les étés alors que son mari reste à la maison. Le milieu bourgeois de lépoque pose un œil admiratif devant ses connaissances, mais se méfie de sa liberté d'agir, de sa vie bourgeoise mais pourtant non traditionnaliste. Sans jamais militer pour la cause des femmes, Jeannette Boulizon vit cette liberté comme si cette dernière allait de soi, dans sa vie de couple, de famille, dans son travail et dans sa vie sociale. Il semble que son statut d'immigrante française pratiquant un métier lui ait donné une crédibilité, une assurance et des possibilités peu habituelles avant la révolution féministe au Québec.

Son mari, Guy Boulizon, est un acteur reconnu du milieu du livre1. Il a tour à tour été professeur, écrivain², critique littéraire3, directeur de la collection «Contes et aventures " créée en 1944 par Fides, premier directeur de la Librairie Flammarion Paris-Montréal limitée en 1950, directeur des Éditions Beauchemin de 1952 à 1964, président de l'Association des écrivains pour la jeunesse, membre du Conseil supérieur de léducation,

1. Né à Nevers en France en 1906, il est décédé à Montréal en 2003.

2. Guy Boulizon a écrit de nombreux livres pour enfants dont Les contes du Mont Tremblant. 1958. Montréal: Beauchemin.; La Croix chez les Indiens. 1958. Montréal: Beauchemin.; Fééries radiophoniques d'après les Mille et une nuits. 1946. Montréal : Fides.

3. Guy Boulizon a dirigé une anthologie littéraire en 1959 chez Beauchemin, mais il est surtout connu pour Livres roses et séries noires, un guide psychologique et bibliographique de la littérature jeunesse paru également chez Beauchemin en 1957. 


\section{Son rôle d'enseignante porte Jeannette Boulizon à partager ses impressions de lecture et à former des lecteurs.}

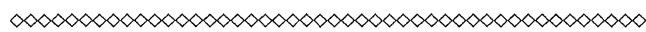

président du Comité consultatif du livre au ministère des Affaires culturelles et collaborateur à différentes revues et émissions radiophoniques, dont Vie des arts et "Radio-Collège ».

Lorsqu'elle n'est pas auprès de son mari et de ses trois enfants et qu'elle trouve des moments libres après le travail, Jeannette Boulizon donne son temps à plusieurs organismes culturels et sociaux. En septembre 1939, elle devient la cheftaine des louveteaux de la meute Stanislas. Elle constate que dans l'enseignement comme dans le mouvement scout, il n'est « [p] as facile, pour une femme, de s'intégrer à ces milieux d'hommes. Non seulement, une cheftaine ne pouvait être assise, dans une auto, près d'un aumônier, mais, au camp, la tente de labbé devait être à cent pieds minimum de celles des guides ou cheftaines" (Boulizon, 1988: 207). Cette expérience de groupe favorise son adaptation au Québec et lui permet d’en apprendre davantage sur la vie québécoise, dans un contexte particulier 4 . Elle participe aussi aux cours de l'École des parents de Montréals. En plus de signer la chronique hebdomadaire de ce regroupement dans Le Devoir, elle en devient la présidente dans les années 1950 et y côtoie un milieu sympathique et accueillant. Selon elle, ce sont des "gens qui pour leur époque sont des animateurs culturels très avant-gardistes " (Boulizon, 1988: 145) et parmi lesquels on retrouve lécrivain Maurice Gagnon, le collectionneur d’art Gérard Lortie et les syndicalistes Simone et Michel Chartrand. Elle joint aussi les rangs de l'Union française, une association qui facilite l'intégration des Français à Montréal ; elle en devient la vice-présidente dans les années 1970. À la même période, elle s'investit dans la communauté chrétienne St-Albert-Le-Grand, une paroisse extra-territoriale gérée par des laïcs qui a, entre autres, le mandat de soutenir les personnes dans le besoin. Elle participe ainsi à la vie sociale en se préoccupant à la fois des enfants et des adultes de son pays d'adoption comme de ceux de son pays d'origine. Elle fait partie de plusieurs réseaux sociaux et culturels et occupe des postes décisionnels dans ces organismes. Elle sait aussi être critique devant

4. Dans Stanislas, un journal à deux voix (1938-1950), le couple Boulizon affirme que c'est par le mouvement scout qu'il a établi un contact réel avec les enfants québécois : "Ce sera grâce aux meutes, aux troupes, au clan, que nous pourrons connaître des jeunes Québécois sous un jour différent de la vie scolaire. Un tel, noté "mauvais élève", sera un scout exceptionnel. Tel autre, fort en thème, fera un piètre campeur. Autre milieu, autres valeurs. " p. 195 et 201.

5. Fondée par Claudine Vallerand en 1940. Ce regroupement apporte de l'aide aux parents par ses forums, ses cours, ses congrès et sa chronique hebdomadaire dans Le Devoir de 1948 à 1955. les actions de ces associations. Par exemple, en 2006, à lâge de 91 ans, elle écrit un message dans le bulletin électronique de St-Albert-Le-Grand et dénonce les membres de sa communauté qui délaissent les paroissiens affligés : "Où est-elle cette communauté chaleureuse, si modèle, qui se démarque tellement des autres paroisses?" (Boulizon, 2006). Jeannette Boulizon est une femme qui n'a pas peur de déranger, de dire ce qu'elle pense et de trouver les bons moyens pour diffuser ses idées.

Responsable de la classe de huitième année au Collège Stanislas dès son arrivée au Canada, Jeannette Boulizon est en contact avec les jeunes et leur enseigne tout, des mathématiques au français en passant par l'histoire et la géographie. Elle leur transmet évidemment les connaissances requises pour réussir le programme français, mais leur fait aussi découvrir des textes et des auteurs du Québec, comme en fait foi le recueil de poèmes et de comptines qu'elle publie avec son mari chez Beauchemin en 1955, Poésies choisies pour les jeunes, où "les inédits d'auteurs canadiens voisinent avec des textes célèbres d’auteurs français " (Boulizon, 1955 : 7). On y retrouve des poèmes de Maurice Carême, de Victor Hugo et d'Alphonse de Lamartine aux côtés de ceux de Simone Routier, de Jean Bruchési et d'Émile Nelligan. Son rôle d'enseignante porte Jeannette Boulizon à diffuser la littérature française et canadienne-française, à partager ses impressions de lecture et à former des lecteurs.

En plus dêtre sa collègue enseignante, Jeannette Boulizon collabore aux projets décriture de son mari, notamment en agissant à titre de première lectrice et en tapant ses textes à la machine. Selon leur fille Michelle (St-Laurent, 2009), Jeannette Boulizon donne son opinion et participe à plusieurs ouvrages, mais son nom napparaît que sur la couverture de trois dentre eux : Poésies choisies pour les jeunes en 1955, Le sapin des premières joies publié aux Éditions du Méridien en 1988 et Stanislas, un journal à deux voix paru chez Flammarion en 1988, dans lequel le couple Boulizon relate ses premières expériences d'enseignants à Montréal. Elle signe aussi, en collaboration avec son mari, la préface du livre de Guy Lapointe Paroles de passage: propos homilétiques aux Éditions Paulines en 1993. Au milieu des années 1970, Jeannette et Guy Boulizon sillonnent ensemble le Québec afin de produire Les musées $d u$ Québec qui sera publié chez Fides mais, bien que louvrage lui soit dédié, le nom de Jeannette n'apparaît pas sur la couverture. Son premier rôle a certainement été d'accompagner son mari dans son métier d’écrivain, de soutenir ses projets et d'y participer à loccasion. D'ailleurs, cette heureuse collaboration a été récompensée, notamment par la Société Saint-Jean-Baptiste qui a décerné le prix Chomedey-de-Maisonneuve en 1989 au couple Boulizon pour ses réalisations ayant contribué au rayonnement de Montréal. Cette position de femme du livre, que l'on pourrait aujourd'hui qualifier de subalterne, mais qui n'en est pas une à l'époque, 
permet à une femme de partager ses idées, de travailler à la réalisation de projets intellectuels et d'apprendre les rudiments du métier décrivain.

À loccasion, Jeannette Boulizon écrit en son nom propre. En 1944, elle se fait critique des nouveautés canadiennes dans le Bulletin des études françaises du Collège Stanislas (Boulizon, 1944). De 1948 à 1955, elle est chroniqueuse au Devoir pour promouvoir les activités de l'École des parents ${ }^{6}$. Elle a écrit un article sur l'histoire de la Société détude et de conférences dans louvrage Ces femmes qui ont bâti Montréal en 1994, puis un article sur le Web qui questionne sa communauté chrétienne en 2006. Sans le concours de son mari, elle n'hésite donc pas à prendre la plume pour partager ses connaissances, pour défendre ce en quoi elle croit et pour le critiquer, s'il le faut.

\section{Femme du livre et regroupements féminins}

À son arrivée au Canada, Jeannette Boulizon constate que de nombreuses femmes de lélite sociale les mères de ses élèves -, bien que très intelligentes, nont pas une culture fort élargie. Lorsquelle veut partager ses impressions de lecture, elle s’aperçoit que les Canadiennes lisent peu et que leurs seules références sont américaines, alors que les siennes sont exclusivement françaises. Les conversations sont donc limitées, ce qui l'attriste énormément (St-Laurent, 2004). À cette époque, le milieu familial accorde peu d'importance à la formation scolaire des filles, même au sein de lélite sociale. Cette situation est commune, car une formation menant à l'exercice d'une profession est pratiquement impensable dans ce milieu. Dans louvrage Mariage et classes sociales, Denise Girard indique quentre les deux guerres, lorsque que les filles atteignent lâge de 18 ans dans la bourgeoisie, les familles accordent beaucoup d'importance à leurs " débuts ", ce moment où les jeunes bourgeoises commencent leurs vies d'adultes en participant aux événements publics mondains. Les femmes, d'abord soutenues économiquement par leurs pères puis par leurs maris, doivent démontrer qu'elles ont de belles manières et qu'elles peuvent tenir maison, sans chercher à développer leur culture personnelle (Girard, 2000). Pourtant, soutient Jeannette Boulizon, "[n]ombreuses étaient celles dont lavidité d'esprit ne pouvait se satisfaire des notions trop rudimentaires du temps. Mesdames Rita Gariépy, Aimée Boucher, Annette Doré-Rochon et toutes les autres assumeront la succession de [1] a fondatrice [de la Société détude et de conférences], Madame Odette Lebrun, avec un enthousiasme qui ne se dément pas soixante ans plus tard. La ferveur qui animait ces jeunes

6. Pour plus d'information sur ce regroupement, on peut notamment consulter le programme de l'année 1950 (Le Devoir, 20 septembre 1950, p. 5). La "Clinique de l'École des parents » est une chronique hebdomadaire qui parait dans la page féminine du Devoir. Des parents en difficulté exposent leurs problèmes auxquels d'autres parents et des spécialistes apportent des pistes de solutions.

\section{Les premiers cercles de femmes, créés au tournant du $X X^{e}$ siècle, témoignent du désir élémentaire d'acquérir des connaissances.}

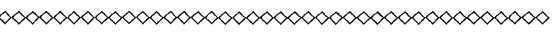

femmes stimulait la curiosité de l'esprit » (Boulizon, 1994 : 292-293).

La Société détude et de conférences est créée dans les années 1930 dans le but de permettre aux femmes de la bourgeoisie de se rencontrer, de réaliser des recherches personnelles et de présenter leurs résultats dans des cercles d'une douzaine de femmes. Affiliée à la Faculté de philosophie de l'Université de Montréal et soutenue par son doyen, le dominicain Marie-Ceslas Forest, la Société rejoint au départ une cinquantaine de femmes de lélite de Montréal. Trente ans plus tard, le mouvement intéresse plus de 1300 femmes et sétend à tous les coins du Québec et de l'est de l'Ontario. La Société demeure en activité encore aujourd'hui.

Pour les regroupements de femmes associés à l'élite, l'importance accordée à la démonstration de la richesse ainsi quà létiquette sont autant de valeurs, de postures et d'attitudes qui déterminent l'appartenance au groupe. L'habitus génère ainsi des pratiques et des comportements associés à la mondanité. "Être mondain, c'est afficher partout, dans ses gestes, ses vêtements, ses paroles, les traits d'élégance, de légèreté, de bon goût, de grande culture que l'on applique [entre autres] à la littérature " (Lacroix, 2004-2005: 63). Être mondain, c'est aussi faire partie d'un cercle de privilégiés qui possède un certain pouvoir symbolique et social. On exhibera chapeaux et voilettes tout comme on invitera des conférenciers français à prendre le thé à l'hôtel Windsor. C'est ainsi que la Société détude et de conférences définira une forme d'esthétique littéraire et mondaine, reposant tant sur son propre capital social que sur celui de ses invités.

Les rôles de tels regroupements correspondent à des besoins particuliers, qui ont évolué avec le temps. Ainsi, les premiers cercles de femmes, créés au tournant du $\mathrm{xx}^{\mathrm{e}}$ siècle, témoignent du désir élémentaire d’acquérir des connaissances. Nombre de cercles et d'amicales qui ont malheureusement laissé peu de traces représentent alors autant doccasions pour les femmes de sortir de leur quotidien, de se rencontrer et d'avoir accès à une forme de culture et de savoir7. D'autres regroupements, développés dans les années 1930, se démarquent des œuvres de charité, de l'artisanat et des multiples formes de rencontres féminines par leur volonté affichée de permettre aux femmes de la bourgeoisie d'accéder à une

7. On pense par exemple au Montreal Local Council of Women (depuis 1893), à la Fédération nationale Saint-Jean-Baptiste (1907-1933) et aux Cercles de fermières (depuis 1915). 


\section{Tableau 1 \\ Conférenciers invités par l'équipe de Jeannette Boulizon entre 1986 et 1989}

\begin{tabular}{|c|c|c|}
\hline $1986-1987$ & $1987-1988$ & 1988-1989 \\
\hline $\begin{array}{l}\text { Hélène Pelletier Baillargeon, journaliste et } \\
\text { auteure }\end{array}$ & $\begin{array}{l}\text { Sœur Thérèse Payer, directrice du Centre } \\
\text { Jeanne-Mance }\end{array}$ & Jeannette et Guy Boulizon, professeurs \\
\hline Hélène Pednault, journaliste et auteure & Antonine Maillet, écrivaine & $\begin{array}{l}\text { Gérard Masson, directeur du Collège } \\
\text { Stanislas }\end{array}$ \\
\hline Robert Prévost, écrivain et historien & $\begin{array}{l}\text { Esther Trépanier, professeure d'histoire de } \\
\text { l'art à l'UQȦM }\end{array}$ & $\begin{array}{l}\text { Germain Lefebvre, conseiller culturel pour } \\
\text { la Communauté urbaine de Montréal }\end{array}$ \\
\hline Jacques Lebreton, écrivain et conférencier & Hubert Reeves, astrophysicien & René Plourde, gemmologue \\
\hline $\begin{array}{l}\text { Raymonde Gauthier, professeure d'histoire } \\
\text { de l'art à l'UQAMM }\end{array}$ & $\begin{array}{l}\text { Waheed Malik, représentant au Québec du } \\
\text { Commissariat aux langues officielles du } \\
\text { Canada }\end{array}$ & Harold Arsenault, réalisateur \\
\hline $\begin{array}{l}\text { Pavel Hamet, professeur de médecine et } \\
\text { directeur de l'Institut de recherches clini- } \\
\text { ques de Montréal }\end{array}$ & $\begin{array}{l}\text { Nadia Magnenat-Thalmann, docteure en } \\
\text { informatique et réalisatrice de films }\end{array}$ & Claire Bonenfant, militante féministe \\
\hline Antoine Padilla, compositeur & $\begin{array}{l}\text { Hubert de Ravinel, consultant en } \\
\text { gérontologie }\end{array}$ & Jacques Drouin, psychiatre \\
\hline Rollande Desbois, professeure & Liliane Blanc, essayiste et auteure & Bohdanna Monczak, enseignante \\
\hline Lily Tasso, journaliste & $\begin{array}{l}\text { François-Marc Gagnon, professeur d'his- } \\
\text { toire de l'art à l'UQAMM }\end{array}$ & $\begin{array}{l}\text { Rolande Allard Lacerte, écrivaine et } \\
\text { journaliste }\end{array}$ \\
\hline Anne-Claire Poirier, cinéaste & $\begin{array}{l}\text { Raymond Beauregard-Champagne, } \\
\text { producteur-réalisateur à Radio-Canada }\end{array}$ & Antoine Padilla, compositeur \\
\hline $\begin{array}{l}\text { Gabriel Villemure, curé de la paroisse } \\
\text { Saint-Léon de Westmount }\end{array}$ & Edgar Fruitier, mélomane et acteur & Doan Trang Phan, actuaire \\
\hline $\begin{array}{l}\text { André Brochu, auteur et professeur de } \\
\text { lettres à l'U. de Montréal }\end{array}$ & Jacques Godbout, écrivain et journaliste & Lesley Lee, mathématicienne et physicienne \\
\hline Michelle Desjardins, professeure & Marthe Beauregard, généalogiste & $\begin{array}{l}\text { Serge Turgeon, président de l'Union des } \\
\text { artistes }\end{array}$ \\
\hline
\end{tabular}

Source : Société d'étude et de conférences. Section de Montréal. Saisons 1986-1987, 1987-1988 et 1988-1989.

culture plus élargie, qui se rapproche des connaissances généralement enseignées dans les collèges classiques. Les regroupements créés dans cette période manifestent un intérêt plus poussé pour l'actualité et leur façon de faire, plus moderne, demandant aux femmes de s'investir personnellement par la recherche et la création, comme cest le cas pour la Société détude et de conférences. Au tournant des années 1960, les regroupements mondains cèdent peu à peu la place à des regroupements d'intérêts sociaux, plus audacieux, plus revendicateurs, précurseurs de la révolution féministe des années $1970^{8}$. Les bouleversements politiques, économiques et sociaux de lépoque de la Révolution tranquille au Québec influencent la sphère culturelle et, inévitablement, les regroupements qui en font partie.

$\mathrm{Au}$ début des années 1940, Jeannette Boulizon enseigne en huitième année à des élèves promis à une brillante carrière9. Les mères de ses élèves, qui ont parti-

8. La Ligue des femmes du Québec (depuis 1957), le Front de libération des femmes du Québec (1969-1971) et le Centre des femmes de Montréal (depuis 1973) sont des exemples de ces lieux de rencontres et de décisions.

9. On trouve entre autres dans sa classe le futur maire d'Outremont, Jérôme Choquette, le futur ambassadeur du Canada à Paris, Michel Dupuy, le futur cinéaste, Claude Jutras, le futur vice-premier ministre du Québec, Jacques-Yvan Morin et le futur premier ministre du Québec, Jacques Parizeau. cipé à la fondation de la Société détude et de conférences, invitent l'enseignante à assister aux conférences dès le début des années 1940 à titre de membre associée, c'est-à-dire qu'elle ne fait partie d'aucun cercle régulier. Dans une entrevue qu'elle maccordait en novembre 2004, Jeannette Boulizon me confiait que certaines de ces femmes l'avaient fortement marquée; elle les a d'ailleurs qualifiées de "surdouées» et considérait notamment Rita Gariépy comme son maitre, l'une des rares femmes au Canada français qui était supérieurement cultivée et avec qui elle pouvait discuter d'égal à égal (St-Laurent, 2004).

Ses obligations familiales, professionnelles et sociales, de même que la faible vacance au sein des cercles plus prestigieux dans lesquels on entre seulement par cooptation, ont pour conséquence de retarder son admission officielle dans un cercle établi. En 1958, elle présente un travail à titre de membre régulier du cercle Sainte-Marie sur Pierre Fontaine, son ancêtre, architecte de Napoléon. Elle prononce une autre conférence en 1962 sur la résurrection de Pompéi. Le cercle dans lequel elle s'investit a été créé en 1945 par Madame Maurice Sainte-Marie, une riche bourgeoise qui a d'importantes relations. Le cercle Sainte-Marie réunit quelques Québé- 
coises et des épouses de consuls en exercice à Montréal. Ces dernières souffrent parfois d'isolement et apprécient échanger avec d'autres femmes sur la situation de leurs pays. Pour les membres réguliers du cercle Sainte-Marie comme Jeannette Boulizon, Nadia Labarre et Geneviève de la Tour Fondue-Smith, c'est une excellente façon de rencontrer d'autres femmes et d'en apprendre davantage sur leurs pays d’origine. C'est aussi pour les Québécoises une occasion de parler de Montréal, des gens qui y habitent, de ce qu'on y fait, de ce qu'on y lit. Les réunions de cercles et les activités régulières de la Société détude et de conférences permettent à Jeannette Boulizon de rencontrer des femmes du monde entier, denrichir sa culture personnelle, de participer aux premiers salons du livre de Montréal au début des années 1950 et d'assister à des conférences offertes par des spécialistes reconnus, de Sartre à Yourcenar en passant par le scientifique Armand Frappier et le musicien Pierre Mercure.

$\mathrm{Ce}$ n'est que tardivement que Madame Boulizon accepte doccuper la présidence de la Société détude et de conférences. Elle a alors 70 ans et veut donner une poussée nouvelle au regroupement, ce qu'elle fait de 1986 à 1989. Elle désire voir la Société détude et de conférences reprendre léclat quoon lui connaissait à ses débuts, notamment en invitant des conférenciers prestigieux comme lécrivain et diplomate Roger Duhamel, la romancière Andrée Maillet, la journaliste Hélène Pednault, lécrivaine Antonine Maillet, l'astrophysicien Hubert Reeves, le comédien Edgar Fruitier et l'écrivain Jacques Godbout, pour ne nommer que ceux-là (Tableau 1). Comme présidente, Jeannette Boulizon constate que les années 1970 et 1980 ont amené les femmes à poursuivre leurs études et à entrer en masse sur le marché du travail. Il faut donc que les dirigeantes de la Société mettent en place de nouvelles mesures pour intéresser les femmes dont les possibilités sont beaucoup moins limitées qu'avant.

En entrevue, Jeannette Boulizon évaluait d'une façon fort éloquente le rôle de la Société détude et de conférences dans la première moitié $\mathrm{du} \mathrm{xx}^{\mathrm{e}}$ siècle :

"Personne ne peut se rendre compte de l'impact qu'a eu la Société d'étude et de conférences. Il est impossible de comprendre aujourd'hui à quel point son influence a été sensationnelle, extraordinaire, historique sur le développement féminin à Montréal, puis à Ottawa, Québec, Chicoutimi et Trois-Rivières. Les femmes qui jusque-là étaient cantonnées à la cuisine et aux enfants, tout d'un coup se sont aperçues qu'elles pouvaient lire, discuter entre elles, écrire et participer à un concours littéraire. Ça a été absolument une révélation. »

(St-Laurent, 2004)

En effet, il existe, dans la première moitié du $\mathrm{xx}^{\mathrm{e}}$ siècle, des regroupements culturels de toutes sortes, culturels, mondains, religieux ou d'anciennes couven-
Si, avant les années 1970 au Québec, les femmes ont peu investi les métiers du livre, elles ont pourtant exercé une influence sur l'histoire culturelle, dont le livre est le vecteur privilégié.

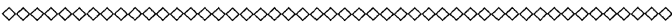

tines, mais aucun de ces groupes ne met à l'avant-plan lacquisition de connaissances, l'accès à une culture générale élargie et approfondie tout en exigeant de ses membres la réalisation de travaux et de recherches. Dans Stanislas, un journal à deux voix, Jeannette Boulizon souligne le caractère novateur du regroupement qui, "sous ses aspects rassurants et bourgeois, est déjà, en fait, un lieu très averti qui, en termes choisis, exprime des idées nouvelles, graves, dérangeantes et suggère des initiatives culturelles hardies" (Boulizon, 1988: 151). C'est certainement pour ces raisons que Jeannette Boulizon a été séduite par la Société d'étude et de conférences et qu’elle a participé à ses activités pendant plus de 60 ans.

\section{Conclusion}

Si, avant les années 1970 au Québec, les femmes ont peu investi les métiers du livre, elles ont pourtant exercé une influence sur l'histoire culturelle, dont le livre est le vecteur privilégié. Leurs actions souterraines, au sein des regroupements, est partie prenante de l'histoire du livre. Létude des regroupements littéraires et culturels permet notamment de saisir l'importance de la mondanité et son lien étroit avec le domaine des lettres. En utilisant la perspective des femmes pour appréhender l'histoire du livre, on réfléchit non plus en fonction des catégories de métiers, mais selon les individus et les réseaux qui y sont rattachés. Ainsi, Jeannette Boulizon peut être considérée comme un exemple dont l'influence auprès de son mari, du milieu du livre et du milieu bourgeois a été remarquable. Ses rôles d'enseignante à Stanislas, d’auteure en collaboration avec son mari ou en solo ainsi que de membre et d'administratrice d'organisations religieuses, éducatives et culturelles, en font une divulgatrice de culture importante puisque ses actions ont pu atteindre plusieurs couches de la société. Son passage à la Société détude et de conférences, par exemple, des années 1940 jusqu'au début du $\mathrm{xxI}^{\mathrm{e}}$ siècle, lui a certainement permis de mieux s'intégrer dans son pays dadoption et de partager son savoir avec son milieu. Femme d'action et de réflexion, Jeannette Boulizon s'est éteinte en 2007, à la fin d'un parcours pluridirectionnel qui l'a amenée à élargir et à partager sa culture personnelle. (-) 


\section{Sources consultées}

Arbour, Roméo. 2003. Dictionnaire des femmes libraires en France, 1470-1870. Genève : Droz. Coll. Histoire et civilisation du livre, 26.

Bettinotti, Julia (dir.). 1990. La Corrida de l'amour : le roman Harlequin. Montréal : XYZ. Coll. Études et documents.

Boulizon, Guy. 1976. Les musées du Québec. Montréal : Fides. Coll. "Loisirs et culture ».

Boulizon, Jeannette. 1944. Madones canadiennes, par Rina Lasnier et Marius Barbeau. Bulletin des études françaises, $\mathrm{n}^{\circ} \mathbf{2 2}$, novembre-décembre : 79-80.

Boulizon, Jeannette. 1994. Société d'étude et de conférences, dans Maryse Darsigny et al. Ces femmes qui ont bâti Montréal. Montréal : Éditions du Remue-ménage. p. 292-293.

Boulizon, Jeannette. 1999. Allocution de Mme Boulizon lors du souper du $60^{\mathrm{e}}$ tenu le 9 avril 1999. <http://ancienstan.com/ historique.htm> (consulté le 2 avril 2010).

Boulizon, Jeannette. 2006. Appel de Jeannette. Bulletin Étapes de la communauté chrétienne St-Albert-Le-Grand de Montréal. $<$ http://www.st-albert.org/Bulletins> (consulté le 2 avril 2010).

Boulizon, Jeanne et Guy Boulizon. 1955. Poésies choisies pour les jeunes. [Montréal] : Beauchemin.

Boulizon, Jeannette et Guy Boulizon. 1988. Stanislas, un journal à deux voix, 1938-1950. Paris : Flammarion.

Darnton, Robert. 1992. Gens de lettres, gens du livre. Paris : Odile Jacob. Coll. Points. Traduction Marie-Alyx Revellat.

\section{Série portant sur I'héritage militaire canadien}

Les anciens combattants sont mieux placés que quiconque pour faire le récit véridique des batailles des deux guerres mondiales et de celle de Corée, car ils ont eux-mêmes vécu la guerre.

Au moyen de documentaires primés à l'échelle internationale, de documents vidéo musicaux et de mémoires, l'Association des Amputés de guerre donne vie aux expériences de volontaires canadiens qui ont servi lors de ces conflits, et rend hommage à ceux qui ont fait le sacrifice de leur vie.

Pour obtenir plus de renseignements ou pour commander des exemplaires, communiquez avec l'Association

des Amputés de guerre ou visitez son site Web, dans lequel figure la liste des titres qui sont offerts.

De précieuses ressources pour les écoles et les bibliotheques $1800250-3030$ amputesdeguerre.ca

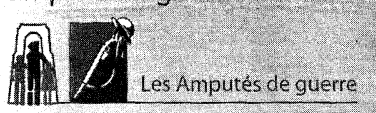
Les Amputés de guerre
Galarneau, Claude. 1978. Les collèges classiques au Canada français. Montréal : Fides.

Girard, Denise. 2000. Mariage et classes sociales. Sainte-Foy: Éditions de l'Institut québécois de recherche sur la culture. Coll. Culture et société.

Lacroix, Michel. 2004-2005. "Toi qui me vois mondaine”. Poésie, mondanité, et écriture des femmes : Les tentations de Simone Routier. Québec Studies, vol. XXXVIII : 59-69.

Lamonde, Yvan et Sophie Montreuil. 2003. Lire au Québec au XIX ${ }^{e}$ siècle. Montréal : Fides.

Lebrun, Monique (dir.). 2004. Les pratiques de lecture des adolescents québécois. Sainte-Foy: Multimondes.

Leroux, Éric. 2005. Histoire de l'imprimerie au Québec. Sherbrooke : Ex Libris. Coll. Études sur l'édition.

Lévesque, Albert et Pierre Dagenais. 1936. La Canadienne française et les lettres. Almanach de la langue française : 55 .

Michon, Jacques. 1999 et 2004. Histoire de l'édition littéraire au Québec au $x X^{e}$ siècle, vol. I : La naissance de l'éditeur (19001939), vol. II : Le temps des éditeurs (1940-1959). Montréal : Fides.

Roy, Fernande. 2000. Histoire de la librairie au Québec. Montréal : Leméac.

St-Laurent, Fanie. 2004. Entrevue avec Jeannette Boulizon, Montréal, 19 novembre 2004, (42 minutes).

St-Laurent, Fanie. 2009. Entrevue téléphonique avec Michelle Prat Boulizon, Montréal, juin 2009.

Tombeur, Jef. 2004. Femmes et métiers du livre. Paris/Soignies : Convention typographique/Talus d'approche. Série « $t$ ». 